\title{
"BOILING THE VACUUM": IN SILICO PLASMAS UNDER EXTREME CONDITIONS IN THE LABORATORY AND IN ASTROPHYSICS
}

• Luís O. Silva - Instituto Superior Técnico, Lisbon - DOI: https://doi.org/10.1051/epn/2017506

Laser technology has progressed tremendously since Theodore Maiman first demonstrated the laser in 1960. One of the most striking examples of this progress is the focused intensity of lasers. Present day lasers in the near infrared frequency range can deliver intensities in excess of $10^{23} \mathrm{~W} / \mathrm{cm}^{2}$. Imagine that you have a very large and powerful lens that captures the light of the sun hitting the upper atmosphere of the Earth and focus it down to a region with the cross section of a human hair. The corresponding intensity is in the range of the most intense lasers now available.

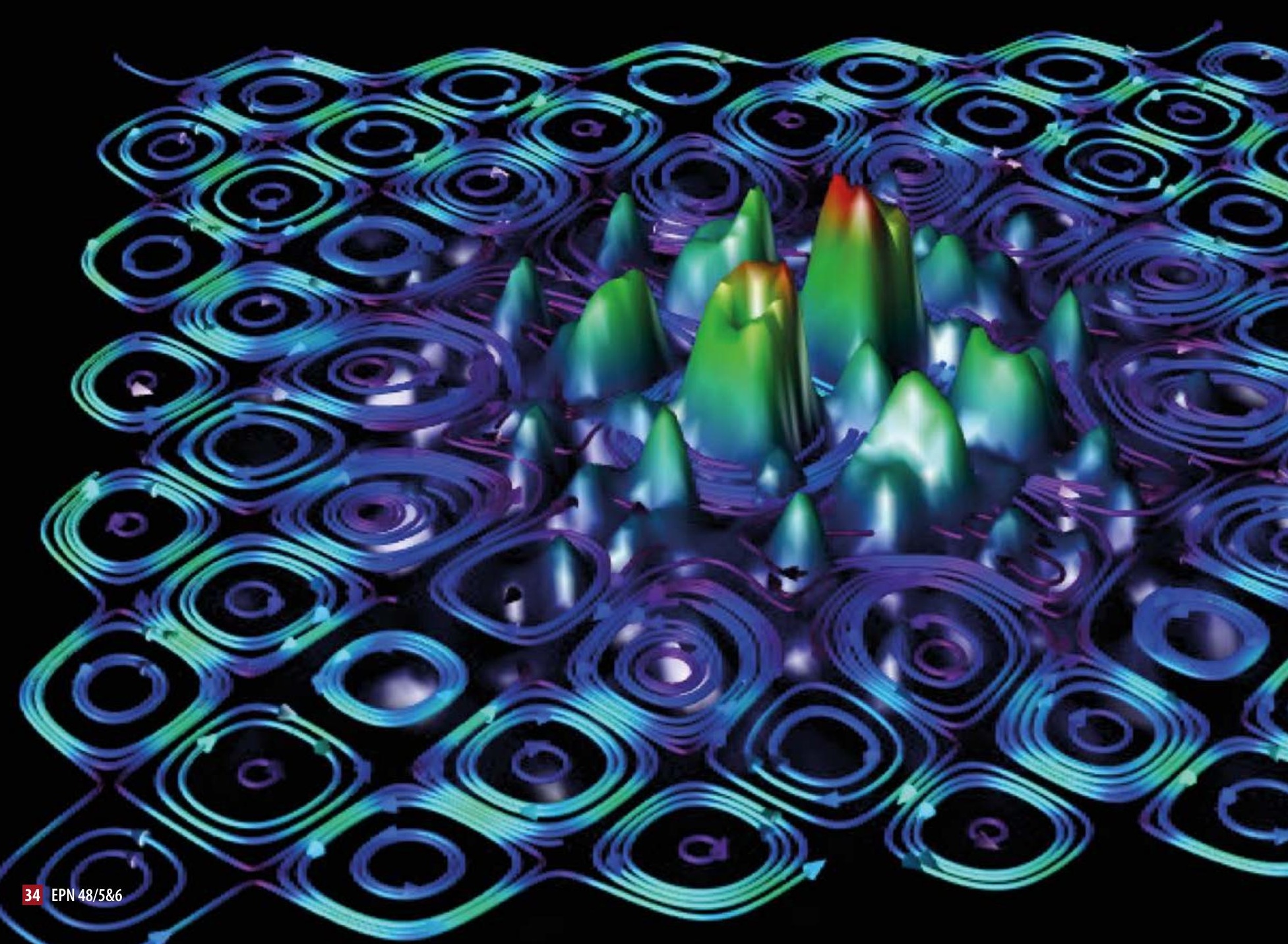




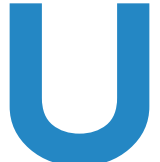

nder the action of these intense electromagnetic fields, electrons quiver at velocities close to the speed of light with Lorentz gamma factors $\sim 100$. The nonlinear effects associated with special relativity determine the evolution of light-plasma interactions at these intensities. These lasers are now being explored to drive plasma accelerators [1,2], or novel light sources [3].

The interplay between lasers, experiments, simulations, and theory is well illustrated by the progress on laser-plasma interactions. Ab initio simulations have been at the core of plasma physics since the 50s, and this is even more striking in laser-plasma accelerators (figure 1): simulations, viewed as in silico experiments, have been critical to interpret and to design many landmark experiments on laser-plasma accelerators, and to predict the directions for new facilities [4].

\section{"Boiling the vacuum"}

Even more intense lasers will be available in the near future, such as the Extreme Light Infrastructure (ELI) [5]. As we move to even higher intensities the physics becomes more rich and more complex [6], and quantum effects

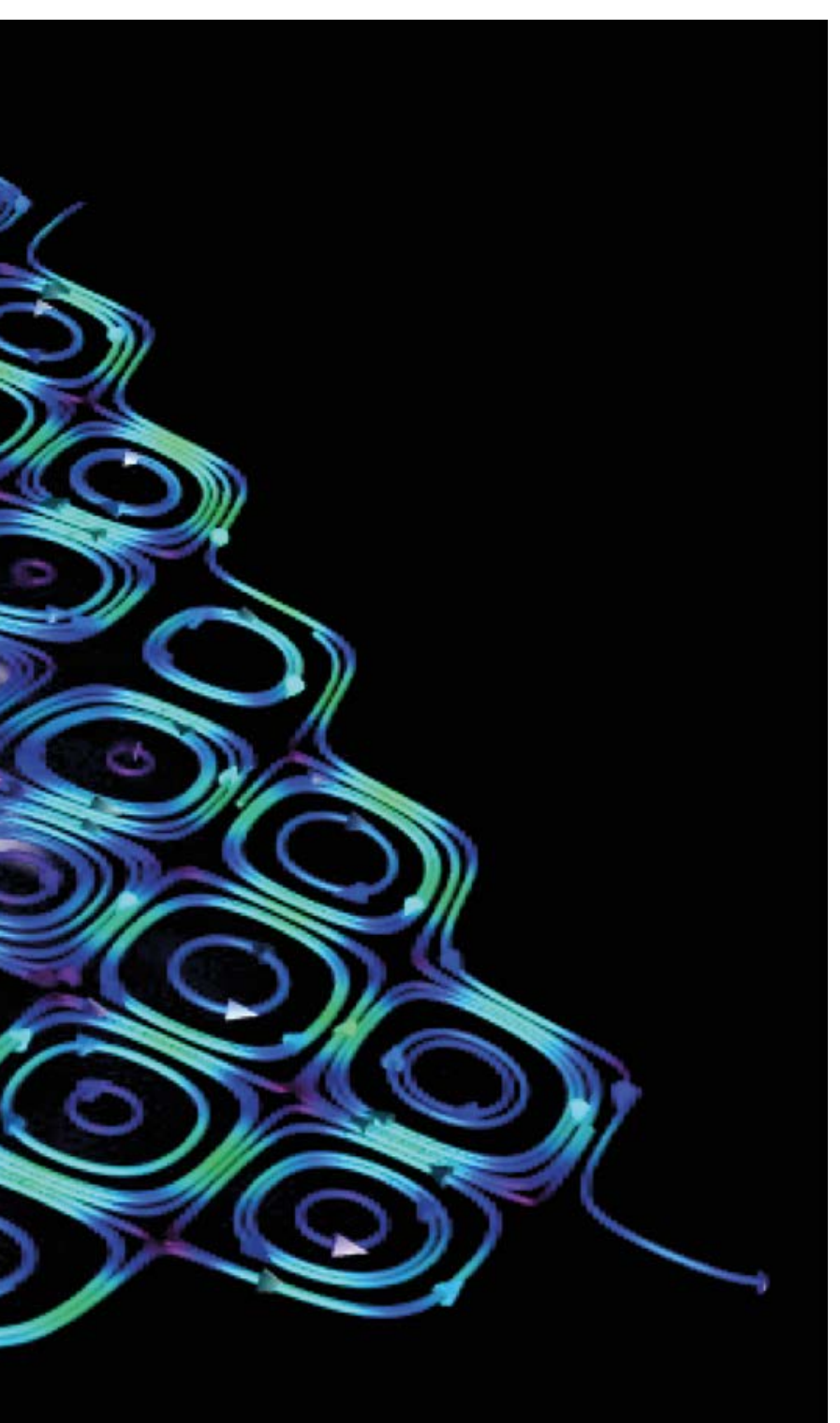

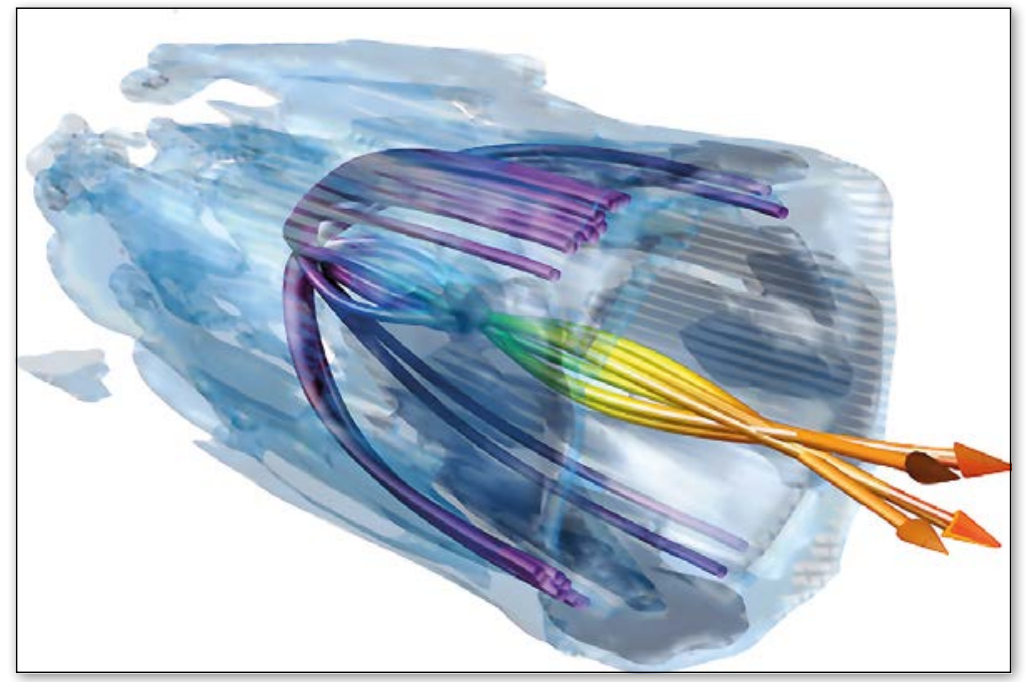

$\triangle$ FIG. 1: The accelerating structure in a laser-plasma accelerator (iso-surfaces), also with the trajectories of trapped and accelerated electrons, from low energies (blue) to high energy (red). Courtesy: Samuel F. Martins

start to be important. A single ultra relativistic electron quivering in an ultra intense laser field can radiate $\mathrm{x}$-rays. As the intensity is ramped up, the electron oscillating in the laser field will radiate gamma-rays. These gamma-rays can, in turn, interact with the laser and generate electron-positron pairs which, in the presence of the laser field, can again radiate strongly. An electromagnetic cascade of pairs and gamma-rays is then triggered, generating a quantum electrodynamic (QED) electron-positron plasma.

It is also possible to conceive scenarios where a static (or slowly varying) ultra strong electric field is present in a finite region of space. Let us imagine the field is so strong that, as the electron is accelerated over the distance of a Compton wavelength it gains more than $2 \mathrm{~m}_{\mathrm{e}} \mathrm{c}^{2}$, i.e. the rest mass energy of an electron and a positron. In this case, an electron-positron pair is generated, which will then interact with the ultra strong field itself, and a cascading process can also be triggered. The critical electric field for pair creation in vacuum, first identified by Sauter, is called the Schwinger critical field $\mathrm{E}_{\mathrm{s}}=2 \pi \mathrm{m}_{\mathrm{e}}{ }^{2} \mathrm{c}^{3} / \mathrm{e} \mathrm{h}$, where $e$ is the electron charge, $m_{e}$ the electron mass, $h$ is the Planck constant, and c the speed of light. For extreme field physics a common used dimensionless parameter is $\chi=E / E_{s}$, where $E$ represents the electric field in the rest frame of the electron. $\chi$ determines the transition from the classical to the quantum dynamics; for $\chi$ on the order or higher than unity, QED effects are important and must be considered. Colloquially, when copious amounts of electrons and positrons are produced by ultra strong electromagnetic fields in vacuum, or via a cascade from a low density seed of electrons, the "vacuum is boiling".

The prospect to reach in the laboratory these conditions in the near future is triggering many exciting developments $[7,8]$ at the cross roads of nonperturbative QED, plasma physics, and astrophysics. In many extreme astrophysical objects (e.g. pulsars, magnetars, or in the magnetosphere $\triangleleft 0$ ptical trap: an efficient converter of light to matter. Four lasers interact with a thin wire of matter (100x thinner than wire is trapped in the vortexes of the electric field and radiates gammarays that decay into electron-positron pairs. These pairs are also trapped and The process continues until most of the laser energy converts to matter (or gammarays). Courtesy: Marija Vranic a human hair). The radiate gamma-rays. 


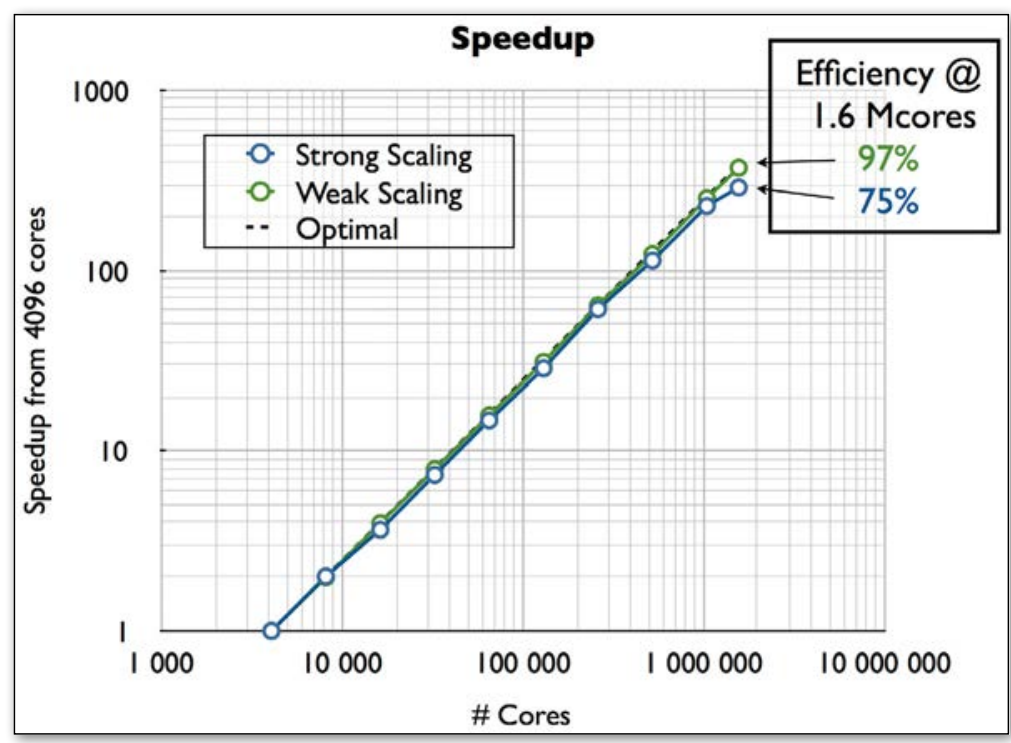

$\triangle$ FIG. 2: Strong and weak scaling of OSIRIS, a state-of-the-art PIC code, for a typical production run in the largest supercomputer in the world as of the time of the benchmark (2013) showing the measured speed-up as a function of the number of processors up to more than 1 million cpu cores. Courtesy: Ricardo A. Fonseca

of black holes), pair production, electromagnetic cascades, and strong radiation reaction are common processes that have an important impact on the properties of the emitted radiation. Therefore, understanding the interplay between the fields and the particles is very important. This renewed interest has also been an opportunity to revisit seminal foundational works, such as the Heisenberg-Euler semi-classical extension of Maxwell's equations, or the Bohr postulate on the maximum possible physically achievable electromagnetic intensity (see [9] for a discussion).

\section{In silico QED plasmas}

The underlying physics is highly nonlinear and depends on the complicated and self-consistent trajectories of individual charged particles in extreme electromagnetic fields, including strong radiation dynamical effects, pair

v FIG.3:OSIRIS simulation (the first ever) of three-dimensional QED cascades. Two laser pulses of opposite circular polarisation collide, showing the cascade (electrons - red, positrons - green, gamma rays - yellow) developing in the plane perpendicular to the laser propagation direction. Courtesy: Thomas Grismayer

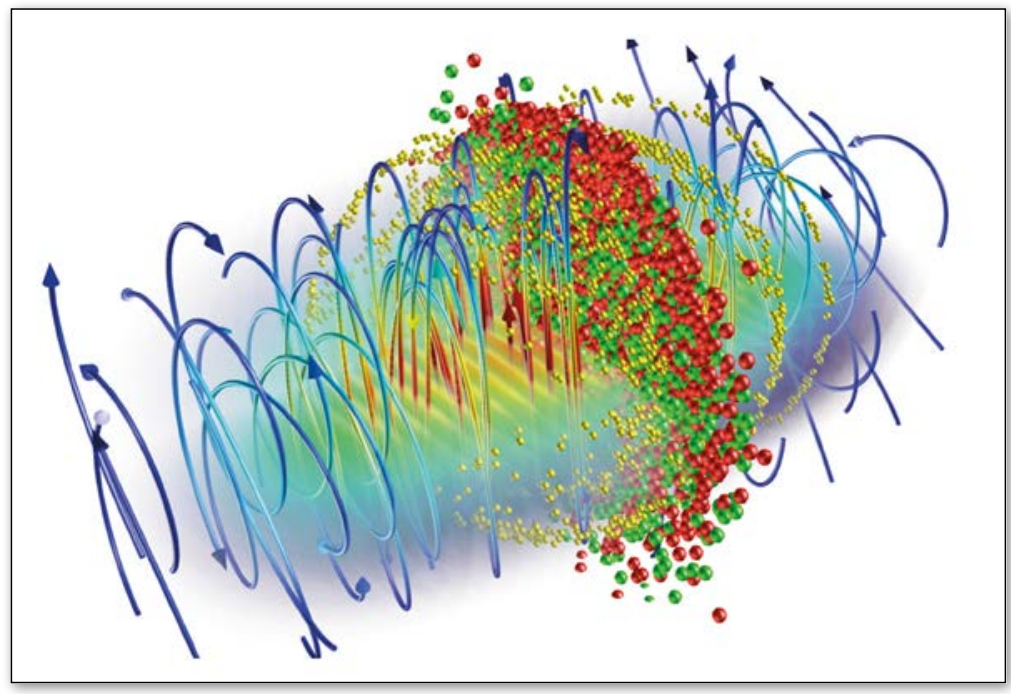

production via QED processes, and, as soon as the cascades develop or if a tenuous plasma is present, collective plasma effects in complex field structures.

Although simplified analytical treatments are possible, the complexity of the systems can only be captured with computational modelling. The exponential developments of high-performance computing have set "computer simulations" as the third pillar of the scientific method, complementing theory and experiments/observation. For QED electron-positron plasmas in intense fields, where laboratory experiments have limited reach, the computational component has enhanced relevance, as a probe of the dynamics of these complex systems, as a tool to identify laboratory scenarios where bridges with the physics of extreme astrophysical environments can be established, or as a tool to model the global dynamics of astrophysical objects and make connection with observations. Therefore, the holistic comprehension of these complex scenarios requires ab initio fully kinetic simulations, following the trajectories of individual particles, complemented by the relevant QED processes.

Plasma physics has a long tradition of kinetic simulations: one of the first dynamical time dependent physical models explored in computers was the one dimensional plasma model, which opened the way to the particle-in-cell (PIC) model [10]. The PIC model is one of the most important computational tools in plasma and one of the most effective possibilities to follow the self-consistent dynamics of charged particles in electromagnetic fields. In the PIC method the trajectories of a large number of particles are solved self-consistently using forces calculated from field equations solved on a grid. When modelling plasmas, the field equations are the Maxwell's equations for the electric and magnetic fields. PIC codes are also used to study a broad set of topics, including accelerator physics, cosmology, and semi-classical systems. The numerical codes based on the PIC algorithm are routinely used in some of the largest supercomputers in the World (figure 2), and thus can fully take advantage of the transformative nature that numerical simulations are bringing to the $21^{\text {st }}$ century science.

It is not surprising then that a generalization of this algorithm has been recently proposed [11-13] to explore the extreme scenarios associated with the coupling of QED with plasmas. These models are computationally very demanding because the plasma scale is coupled with the QED scale via a probabilistic event generator technique. The possibility to take advantage of Peta floating point operations per second (Petaflop/s) supercomputers has already unveiled, even in the most simplified settings, the richness of the physics, ranging from radiation reaction effects in the collision of electron beams with lasers, to the self-consistent evolution of electromagnetic pair-plasma cascades, or the optimal configurations for electromagnetic cascades 


\section{Exploring extreme plasma conditions in the lab and in astrophysics}

$A b$ initio simulations are already capturing the multidimensional collision of multi $\mathrm{GeV}$ electron beams with multi petawatt laser systems [14], showing this is an ideal setting to explore the transition from classical radiation reaction to quantum radiation reaction, associated with the transition of a regime with low $\chi$ to $\chi \sim 1$, opening the way to test different models for radiation reaction.

Recent three dimensional simulations have also allowed to determine the conditions for electromagnetic cascades with ELI scale lasers [15], including the radiation signatures and the properties of the associated gamma ray source, and the dependence of these processes on the configurations of the laser, taking into account the polarization or multi-beam configurations.

From a plasma physics point of view, the interplay between collective processes and QED effects associated with very high fields is relevant in high charge electron beam-electron/positron beam collisions, at the interaction point, when beam disruption can be important. Collective plasma processes are also important when the density of the self-generated plasma due to the QED cascade is so high that the plasma becomes opaque to the laser itself. In this case there is strong light absorption and efficient conversion of energy from the laser to the pair plasma and the gamma rays [13].

What is more exciting is that many of the processes that we are now exploring and understanding are at the core of some of the most exciting astrophysical objects. In magnetars, magnetic fields with magnitude close to the Schwinger field are present and the interplay between the fields, the plasma, and the QED processes remains an open question. In the magnetosphere of pulsars, electromagnetic cascades are also thought to be relevant. Global models for the magnetospheres of pulsars have been recently implemented but by coupling QED effects with the global geometry for the fields in rotating magnetospheres, as illustrated in Figure 4 , it will be possible to understand the global dynamics of the system, determine radiation signatures, and identify the physical processes that determine the radiation signatures to mimic and further explore in the laboratory these physical processes, thus understanding how the "vacuum boils" in the laboratory and in astrophysics.

\section{About the Author}

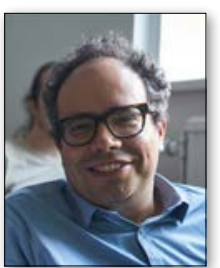

Luís O. Silva is Professor of Physics at Instituto Superior Técnico, Lisbon, PhD and Habilitation from IST, and post-doc at UCLA (1997-2001). He was awarded two ERC Advanced Grants, among other prizes/honors. He is Fellow of the APS, Global Young Academy, and the EPS. http://web.tecnico.ulisboa.pt/luis.silva/ http://epp.tecnico.ulisboa.pt/

\section{Acknowledgments}

I would like to thank R. Fonseca, T. Grismayer, M.Vranic, F. Cruz, and everyone at the epp team at IST for discussions. Work partially supported by the European Research Council AdG2015 InPairs.

\section{References}

[1] C. Joshi, Scientific American 294 (2), 40 (2006)

[2] W. P. Leemans and E. Esarey, Physics Today 6 (3), 44 (2009)

[3] K. Ta Phuoc et al., Nature Photonics 6, 308 (2012)

[4] S. F. Martins et al., Nature Physics 6 (4), 311 (2010)

[5] https://eli-laser.eu

[6] G. A. Mourou, T. Tajima and S. V. Bulanov, Reviews of Modern Physics 78 (2), 309 (2006)

[7] M. Marklund and P. K. Shukla, Reviews of Modern Physics 78 (2), 591 (2006)

[8] A. Di Piazza et al., Reviews of Modern Physics 84 (3), 1177 (2012)

[9] A. M. Fedotov et al., Physical Review Letters 105, 080402 (2010)

[10] J. M. Dawson, Reviews of Modern Physics 55 (2), 403 (1983)

[11] E. N. Nerush et al., Physical Review Letters 106, 035001 (2011)

[12] C. P. Ridgers et al., Physical Review Letters 108, 165006 (2012)

[13] T. Grismayer et al., Physics of Plasmas 23 (5), 05670 (2016)

[14] M. Vranic et al., Physical Review Letters 113 (13), 134801 (2014)

[15] T. Grismayer et al., Physical Review E 95 (2), 023210 (2017)

V FIG.4:Ab initio simulation of a rotating magnetosphere showing the field lines of a centrifugally-driven magnetosphere, with the spiralling features already present in the density, with plasma uniformly loaded in the simulation region (projection in the top of the simulation box) Courtesy Fabio Cruz

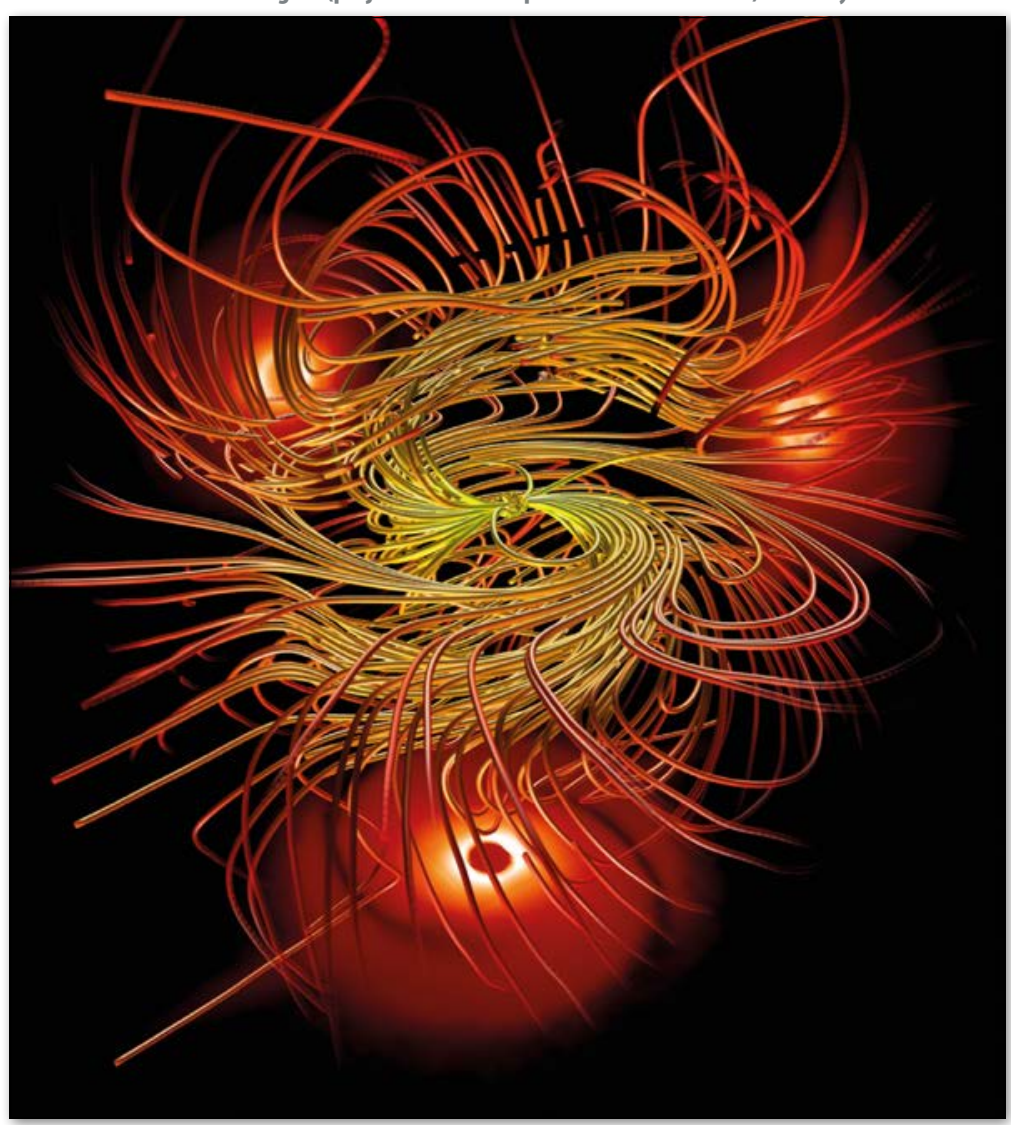

